\section{Collective papers in cognitive psychology}

Thinking: Readings in Cognitive Science. Edited by P. N. Johnson-Laird and P. C. Watson. Pp. 615. (Cambridge University Press: Cambridge, New York and London, 1977.) Hardback £17.50; paperback $£ 5.95$.

THE term Cognitive Science is used by the editors of this volume to refer to the new approach to "the scientific study of thinking" which has emerged from the cross-fertilisation, debates and occasional collaborations between cognitive psychology, linguistics and artificial intelligence (AI) during the past decade. The hallmark of the approach is its espousal of process models of human behaviour: postulated information-processing structures, typically cast in the form of computer programs, which suffice to generate the phenomena of interest. This approach contrasts sharply with the more traditional methodology in cognitive psychology which sees as its role the more functional task of establishing empirically what factors affect performance and in what ways. Questions about the underlying mechanism, if addressed at all, are discussed in terms deriving from neurophysiology rather than information processing.

The book contains 34 readings (three of them written specially) divided into seven sections: Problem Solving; Deduction; Conceptual Thinking; Hypotheses; Inference and Comprehension; Language, Culture and Thinking; and Imagery and Internal Representation. Each section begins with an extensive introduction to the topic and there is a ten-page Introduction to the book as a whole. Despite its interdisciplinary stance, in practice the book draws mainly from sources in cognitive psychology with occasional contributions from AI, philosophy and anthropology. Some 23 of the papers are by authors whose primary allegiance is to psychology and 6 are by people in AI (including one example of genuine collaboration across disciplines), lcaving 5 from other fields. To the extent that it is of value to speak of 'themes' running through such a collection of papers, the most striking have to do with the pervasive role of inference in language comprehension and the difficulty people have in trying to draw conclusions from negative information. A secondary refrain concerns the fragile basis of Man's claim to rationality.

Whatever one feels about some of the individual choices, there can be no doubt that the collection as a whole is a stimulating one. Some of these papers have become minor classics, either because of their seminal quality (Shepard and
Metzler's original paper on mental rotation of objects, Huttenlocher's and H. H. Clark's contrasting analyses of threeterm series problems) or because of their concise summary of an area of investigation (for example, Tversky and Kahneman's catalogue of human fallibilities in handling probabilistic information). The Genevan viewpoint is represented by extracts from a paper by Piaget dealing with intellectual development from adolescence to adulthood, and by a delightful piece by Karmiloff-Smith and Inhelder in which the careful, insightful observation and description of children's attempts to balance wooden blocks provides a salutary example to workers in $\mathrm{AI}$ and in mainstream cognitive psychology alike.

This collection was assembled specifically to meet the needs of students taking a new Open University course on cognitive psychology. It will be of relevance to all those who are interested in the impact that advances in linguistics and AI have had on psychologists' view of cognition. However, the book is not

\section{Superradiance rules the day}

Cooperative Effects in Matter and Radiation. Edited by C. M. Bowden, D. W. Howgate and H. R. Robl. Pp. 388. (Plenum: New York, 1978.) \$45.

R. H. Dicke's famous (and obscure) paper on cooperative spontaneous emission of radiation, or "superradiance", was published twenty-four years ago. The present volume, representing the proceedings of a cooperative effects meeting at Redstone Arsenal, Alabama, in late 1976 consists for the most part of descriptions of various approaches to superradiant theory and experiments. The word superradiant has often been misused (even incorrectly applied to high-gain mirrorless lasing) but is now taken to mean either the coherent radiation generated by a system of atoms possessing an externally created macroscopic polarisation rather akin to a phased array of dipoles, or alternatively the incoherent fluctuating radiation produced by a system of excited atoms possessing no initial polarisation but instead developing a coopcrative decay behaviour through quantum correlations. The word "superfluorescence" has been proposed by Rodolfo Bonifacio for this latter process which can be distinguished by its coherence properties and its directionality. The explosion of theoretical interest in the late 1960s and early 1970s in superradiance has been followed very recently by a number of experiments on far-infrared superradiance, described without its defects. Apart from typographical errors, a couple of the papers have been 'edited' to the point of nearincomprehensibility. The distinctions between some of the seven sections are unclear. And the reader should be forewarned that the particular view of cognitive science which shapes the book is very much the editors' own. The emphasis is heavily biased towards the linguistic aspects of cognition, with other areas being painfully under-represented. In several places the editors come close to identifying 'thinking' with (verbal) 'reasoning', and when reading the introductions to each section this reviewer had several times to re-assure himself that he had not accidentally picked up a textbook on psycholinguistics. These Readings need to be supplemented by other readings to provide a balanced picture of modern thinking about thinking.

Richard M. Young

Richard Young is an SSRC Fellow in the Department of Artificial Intelligence at the University of Edinburgh, Scotland.

here by Gibbs, Vrehen and others.

Leading problems in superradiance are whether or not single-pulse emission or "ringing" occurs, and how the initial coherence is established. The two leading theoretical camps are well represented here. The mean-field approach advocated by Bonifacio, with singlepulse emission and with no role being played by propagation, is described by Bonifacio and by Bullough. On the other hand, the computcr-solution approach to the totally semiclassical coupled Maxwell-Bloch equations is described by Mike Feld and emphasises propagation and associated ringing of the pulsed emission. The discussion of the conference papers is published verbatim at the end of this volume and illustrates the heat generated by this topic. The interesting and difficult problem of the initial evolution of the system and the establishment of coherence is indicated as the outstanding difficulty left to solve.

Other papers in this volume are concerned with the free-electron laser experiment at Stanford and the theory behind it, the Dicke phase transition, and self-focussing in self-induced transparency. But superradiance obviously ruled the day. 'This is a stimulating volume, unusual in so clearly depicting the problems remaining to be solved and the conflicts between rival explanations. It is specialised and expensive, but certainly deserves to be in institutional libraries.

Peter Knight

Peter Knight is Jubilee Research Fellow in the Department of Physics at Royal Holloway College. University of London. UK. 